\title{
Development of Methods for Safe Application of Viral Vectors for Production of Gene-Engineering Vaccines
}

\author{
Sainova IV1*, Valkova IP ${ }^{2}$, Markova $\mathrm{T}^{3}$ and Nikolova EB ${ }^{1}$ \\ ${ }^{1}$ Bulgarian Academy of Sciences, Institute of Experimental Morphology, Pathology and \\ Anthropology with Museum, "Acad. Georgi Bonchev" Street, Bl. 25, 1113 Sofia, Bulgaria \\ 21-st Pediatric Consultative Clinic, Sofia, Bulgaria \\ Research Article \\ Volume 2 Issue 7 \\ Received Date: June 13, 2018 \\ Published Date: June 25, 2018 \\ *Corresponding author: Iskra Ventseslavova Sainova, Bulgarian Academy of Sciences, Institute of Experimental \\ Morphology, Pathology and Anthropology with Museum, “Acad. Georgi Bonchev" Street, Bl. 25, 1113 Sofia, Bulgaria, \\ Email: iskrasainova@gmail.com
}

\section{Abstract}

Vaccine avipoxviral strains FK (fowl) and Dessau (pigeon) were adapted for replication in heterologous for them mammalian cells from the embryonic bovine trachea cell line EBTr. A proof for their successful adaptation was the cytopathic effect (CPE) on mammalian cell cultures, expressed in appearance of cells with round shape, cytoplasmic vacuolization and detachment of the cells from the substrate. In application of low initial infections titers of $10^{3}$ $\mathrm{CCID}_{50} / \mathrm{ml}$ (high initial dilutions of $10^{-3} \mathrm{CCID}_{50} / \mathrm{ml}$, respectively) of the viral suspension for both eventually attenuated by many passages from the heterologous for mammals and mammalian cells avian vaccine strains, but no CPE was established. In freezing of the so inoculated with the diluted viral suspensions mammalian cells after addition of cryoprotector Dimethylsulfoxide (DMSO), thawing and re-incubation in fresh cultivation medium, signs of activated cell proliferation (as decreased monolayer density and formation of internal "islands" of cells in the monolayer) were noted. One of the possible explanations could be eventual transfer of nucleotide sequences from viral particles to separate cells because of activated fusion processes on the influence of DMSO in drastic temperature changes. The results obtained proposed a possibility about application of the described methods as available alternatives for production of geneengineering vaccines, and were in confirmation with scientific references. Furthermore, a possibility for production of membrane glycoprotein receptors and other immune molecules from non-immune cells in appropriate conditions (presence of viral particles) was suggested, which was also in agreement with literature findings. Future studies are necessary in this direction, directed mainly to isolation and purification of the eventually received recombinant viral 
vaccines from the cultural fluids of the infected cell cultures, as well as subsequent investigations on their immunogenic potential in vitro and in vivo.

Keywords: Vaccine Viral Strains; Mammalian cells; Nucleotide Sequences Transfer; Activated fusion process; Production of Immune Molecules; Methods for Preparation of Gene-Engineering Vaccines

\section{Introduction}

Many literature data about the role of viruses as appropriate vectors for gene transfer have been obtained for both viruses, which genome is DNA and/or RNA, both in vitro and in vivo, have been proved [1-4]. Messages about application of mixed recombinant DNA-constructs, composed by DNA-fragments have also been obtained containing components from different viral strains [5-7] or from different organism origin (viral, bacteria plasmid, yeast and/or other eukaryotic cell types) $[8,9]$. For this goal, appropriate promoter gene should be inserted, necessary to provide the expression of the respective inserted gene of interest. The other main component is a marker gene, localized to immediate nearness to the tested gene of interest, necessary for selection of the cells, positive of the last. Most often, as marker genes could be used such, which code color proteins (for example, fluorescent protein, as GFP, YFP, RFP) or products, participating in a color reaction (enzymes luciferase or timidinkinase), or is connected in any way resistance to any antibiotic preparation. The described gene sequences should be connected by treatment with specific ligases, but the used initial vector constructs should contain specific restriction sites, which is necessary the withed DNA-fragments of interest be obtained by treatment with respective restriction enzymes (most often bacterial endo-nucleases). Other important components are the reverted end DNA-repeats. Poxviral DNA-genome has been characterized among the most appropriate and applicable material for production of gene-engineering vaccines [10]. On the other hand, the property of the cryoprotector Dimethylsulfoxide (DMSO), as well as of other organic detergents, to activate the fusion processes mainly by changing the properties of the biological molecules, has been proved [11-14].

In this connection, the main goal of the current study was directed to development of methods for maximally safe application of gene-engineering viral vaccines.

\section{Materials and Methods}

Methods for safe applications of avipoxviral strains FK (fowl) and Dessau (chicken) (Poxviridae family) as initial material for design of appropriate vectors for immunization and gene-engineering manipulations with mammals and mammalian cells, were developed. Cells of embryonic bovine trachea were used. EBTr cell line from embryonic bovine trachea, derived by Kniazeff, et al., was used [15]. The cells were incubated in combination of Modified Eagle's Medium (MEM) (Sigma) and Dulbecco's Modification of Eagle's Medium (DMEM) (Sigma), in ration 1:1, supplemented with 5\% mixture of Fetal Bovine Serum (FBS) (Sigma) and Normal Bovine Serum (NMS) (Sigma), as well as of antibiotics mixture of $(100 \mathrm{UI} / \mathrm{ml}$ Penicillin and $100 \mu \mathrm{g} / \mathrm{ml}$ Streptomycin) (Sigma), at $37^{\circ} \mathrm{C}$ in incubator with $5 \% \mathrm{CO}_{2}$ and $95 \%$ air humidification. Three main steps for development of strategy about safe application of both viral strains were evaluated: application of heterologous for mammals and cells from them avian viral strains; of attenuated vaccine forms of both strains by many passages in cell cultures and chicken embryos, as well as of comparatively low initial infections titers of $10^{3} \mathrm{CCID}_{50} / \mathrm{ml}$ viral suspension (high initial dilutions of viral suspensions of $10^{-3} \quad \mathrm{CCID}_{50} / \mathrm{ml}$, respectively) [16-18]. Separated sub-populations of mammalian cells, inoculated with the same low dilutions of the suspensions of both viral vaccine viral strains, were freezed at $-80^{\circ} \mathrm{C}$ or at $-196^{\circ} \mathrm{C}$ (liquid nitrogen) after previous addition of respective volume from the cryoprotector DMSO (Sigma), added directly to the inoculated cell cultures, for 1-2 weeks. After thawing, the so prepared cultures were re-cultivated in the described above incubation conditions. For this aim, all thawed cell suspensions were centrifuged, and after turning-off of the supernatants, the pellets were washed with PBS (Sigma), resuspended, and the cell suspensions received were put in fresh cultivation medium, composed of the gradients, described above. All cell cultures were observed as native preparations, by inverted light microscope, supplied with mega-pixel CCD-camera. 


\section{Virology \& Immunology Journal}

\section{Results}

Cytopathic effect (CPE), expressed by cytoplasmic vacuolization, shape changing (rounding) and detachment of from the substrate, was observed in the cell cultures, infected with undiluted suspensions $\left(10^{0} \mathrm{CCID}_{50} / \mathrm{ml}\right)$ of both vaccine avipoxviral strains (Figures 1B \& C), compared with the control uninfected mammalian cells (Figure 1A). This effect was stronger in the cells, inoculated with the fowl strain FK (Figure 1B) than in these, infected with the pigeon strain Dessau (Figure 1C). In the cell cultures, inoculated with initial dilutions of initial infections titers of $10^{3} \mathrm{CCID}_{50} / \mathrm{ml}$ (high initial dilutions of viral suspensions of $10^{-3} \mathrm{CCID}_{50} / \mathrm{ml}$ ) of both strains (Figures 1D \& E), for the first time CPE was not observed by light microscopy assay and no significant differences in comparison with the control non-infected cells (Figures 2D) were established. In application of 3 lgs lower initial infectious titer of $10^{6} \mathrm{CCID}_{50} / \mathrm{ml}$ (of 3 lgs higher initial viral suspension dilutions of $10^{-6} \mathrm{CCID}_{50} / \mathrm{ml}$, respectively) for both vaccine viral strains, not only no cyto-pathogenic changes were observed, but signs activated cell proliferation in both cases (Figures $1 \mathrm{~F} \& \mathrm{G}$ ) in comparison with the non-inoculated control were noted (Figure 1A), as increased cell density, decreased $\mathrm{pH}$ of the medium in comparison with the cells, inoculated with the non-diluted viral suspensions of both strains (Figure 1B \& C) and formation of internal cellular "islands" in the monolayer. One of the possible explanations was eventual insertion of viral genetic material in the cellular genome of separated cells because of activated fusion process on the influence of cryoprotector DMSO in drastic temperature changes.
A

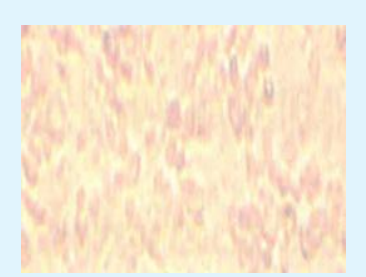

E
B
C

D

Figure 1: EBTr mammalian in vitro-cell cultures, inoculated with vaccine avipoxviral strains FK (fowl) and Dessau (pigeon): A - control non-inoculated culture; B, D, F - inoculated with FK, C, E, G - inoculated with Dessau; B, C - with non diluted viral suspensions of both strains $\left(10^{\circ} \mathrm{CCID} \mathrm{D}_{50} / \mathrm{ml}\right)$; D-F - with dilutions of both strains $10^{3} \mathrm{CCID}_{50} / \mathrm{ml} ; \mathrm{D}, \mathrm{E}-$ non-passed through freezing in the presence of DMSO, subsequent thawing and re-incubation, F, G - passed through freezing in the presence of DMSO, subsequent thawing and re-incubation (Native light-microscopy preparations, magnification: $100 \mathrm{x}$ ).

\section{Discussion}

The proved high self-renewal potential of the stem cells in in vitro-conditions makes them strong candidates for delivering of genes, as well as for restoring organ systems function have been found to be included in these processes $[6,7,9]$. The obtained data were in agreement of many literature data about proved ways for safe applications of avian viral strains on non-permissive for them mammalian cells $[19,20]$. One of the probable explanations about the limited viral replication possibilities could be the non-permissive for avian strains mammalian cells, which is in agreement with the literature data, as well as with these, obtained in our previous studies. The potential of viruses as appropriate vectors for development of various therapeutic strategies has been proved in many literature sources - for both immunization by application of recombinant vaccines and 
therapeutic procedures in different disorders by substitution of appropriate cell nucleotide sequences $[2,3,5,6,21]$. In this connection, the lack of CPE in mammalian cells, inoculated with initial dilutions of $10^{3}$ $\mathrm{CCID}_{50} / \mathrm{ml}$ (with viral suspensions dilutions of $10^{-3}$ $\mathrm{CCID}_{50} / \mathrm{ml}$, respectively), but the presence of immature viral particles, could suggest a possibility about application of the strains in this form as material in vaccine production, including for the goals of the immunotherapy of malignancies. The higher amount of immature virions in cells, inoculated with the vaccine viral strain FK in comparison with this in the cells, infected with strain Dessau, was in support of our previous results about the proved stronger in vitro-CPE, induced by the fowl vaccine strain than the induced by the pigeon vaccine strain, as well as with other literature data $[10,17,18]$. In this way, a possibility for application of the vaccine fowl pox viral strain FK as a usable source for preparation of vaccines for immune-prophylaxis and immune-therapy was supposed, also in agreement with the literature finding about the high immunogenic potential of this strain [21]. On the other hand, the proposed transfer of nucleotide sequences from viral particles to mammalian cells as a result of activated fusion on the influence of DMSO in drastic temperature changes was in support of the reference data about such effects of this cryo-protector, but also of other organic detergents [11-13]. As a main reason, underlining the fusion activation between membrane structures in these conditions, was suggested the changes in the properties of the membrane-building molecules, as well as in the intermolecular interactions, on the influence of some organic substances [14]. Besides the influence of DMSO and of the drastic temperature changes, helping action of the fusion process by activated production of membrane glycoproteins and other immune molecules from nonimmune cells in appropriate conditions (on the influence of the infectious agent), proved in the scientific literature. The last reaction could be explained with mentioned in many literature messages "intrinsic antiviral immunity" changes, as internal protection of the cell in response to its infection [22,23]. Another explanation could be connected with eventual appearance of early signs of immune cell differentiation of immature embryonic cells on the influence of the foreign for them viral particles, which has been shown in several scientific references, as well as in our previous studies [24-28]. Further studies are necessary in this direction, connected with development of methods for isolation and purification of the eventually received recombinant viral vaccines from the cultural fluid of the inoculated cells, as well as subsequent investigations on their immunogenic potential in vitro and in vivo.

\section{References}

1. Boyle DB, Coupar BE, Gibbs AJ, Seigman LJ, Both GW (1987) Fowlpox virus thymidine kinase: nucleotide sequence and relationships to other thymidine kinases. Virology 156(2): 355-365.

2. Cadoz M, Strady A, Meignier B, Taylor J, Tartaglia J, et al. (1992) Immunization of man with canarypox virus expressing rabies glycoprotein. Lancet 339(8807): 1429-1432.

3. Galindo I, Lorenso M, Blasco R (2001) Set of vectors for the expression of histidine-tagged proteins in vaccinia virus recombinants. Biotechniques 30(3): 524-526.

4. Barrette S, Douglas JL, Seidel NE, Bodine DM (2000) Lentivirus-based vectors transducer mouse hematopoietic stem cells with similar efficiency to moloney murine leukemia virus-based vectors. Blood 96(10): 3385-3391.

5. Borysiewicz LK, Fiander A, Nimako M, Man S, Wilkinson GW, et al. (1996) A recombinant vaccinia virus encoding human papillomavirus types 16 and 18, E6 and E7 proteins as immunotherapy for cervical cancer. Lancet 347(9014): 1523-1527.

6. Kent SJ, Zhao A, Best SJ, Chandler JD, Boyle DB, et al. (1998) Enchanced T-cell immunogenecity and protective efficacy of a human immunodeficiency virus type 1 vaccine regimen consisting of consequtive priming with DNA and boosting with recombinant fowlpox virus. J Virol 72(12): 101801088.

7. Panicali D, Paoletti E (1982) Construction of poxviruses as cloning vectors: insertion of thymidine kinase gene from herpes simplex virus DNA of infectious vaccinia virus. Proc Natl Acad Sci USA 79(16): 4927-4931.

8. Domi A, Moss B (2005) Engineering of a vaccinia virus bacterial artificial chromosome in Escherichia coli by bacteriophage $\lambda$-based recombination. Nat. Meth 2(2): 95-97.

9. Leong K, Ramsey A, Boyle D, Robinson H, Ramshaw I (1994) Novel vaccine strategies using fowlpox viruses and plasmid DNA. Ann Conf Australas Soc HIV Med 6: 3-6. 


\section{Virology \& Immunology Journal}

10. Szajner P, Weisberg AS, Wolffe EJ, Moss B (2001) Vaccinia virus A30L protein is required for association of viral membranes with dense viroplasm to form immature virions. J Virol 75(13): 5752-5761.

11. de Ménorvan MA, Mir LM, Fernández LM, Reigada R (2012) Effects of dymethyl sulfoxide in cholesterolcontaining lipid membranes: a comparative study of experiments in silico and with cells. PLoS One 7(7): e41733.

12. Manandhar G, Onishchenko GE (1995) Centriolar cycle of fused cells. J Cell Sci 108(Pt 2): 667-673.

13. Norwood TH, Zeigler CJ, Martin GM (1976) Dimethyl sulfoxide enchances polyethylene glycol-mediated somatic cell fusion. Somat Cell Genet 2(3): 263-270.

14. Jensen MA, Fukushima M, Davis RW (2010) DMSO and Betaine greatly improve amplification of GCrich constructs in de novo synthesis. PLoS ONE 5(6): e11024.

15. Kniazeff A, Nelson Rees W, Darby N (1965) Experta Medica 19 (Section 1).

16. Popova T, Sartmadjiev K (1999) Immunomodulation in chickens immunized against avian pox. Compt rend de'l Acad bulg Sci 51(1-2): 101-104.

17. Sainova IV, Kril AI, Simeonov KB, Popova TP, Ivanov IG (2005) Investigation of the morphology of cell clones, derived from the mammalian EBTr cell line and their susceptibility to vaccine avian pox virus strains FK and Dessau. J Virol Meth 124(1-2): 37-40.

18. Sainova IV (2007) In vitro-differentiation of embryonic mammalian cells as a material base for gene-engineering manipulations. Internal J Bioautomation 8(1): 201-206.

19. Somogyi P, Frazier J, Skinner MA (1993) Fowlpox virus host range restriction: gene expression, DNA replication, and morphogenesis in nonpermissive mammalian cells. Virology 197(1): 439-444.
20. Wazer DE, Liu XL, Chu Q, Gao Q, Band V (1995) Immortalization of distinct human mammary epithelial cell types by human papilloma virus 16 E6 or E7. Proc Natl Acad Sci USA 92(9): 3687-3691.

21. Taylor J, Weinberg R, Languet B, Desmettre $\mathrm{P}$, Paoletti E (1988) Recombinant fowlpox virus inducing protective immunity in non-avian species. Vaccine 6(6): 497-503.

22. Murray MJ, Peters NE, Reeves MB (2018) Navigating the host cell response during entry into sites of latent cytomegalovirus infection. Pathogens 7(1).

23. Yan N, Chen ZJ (2012) Intrinsic antiviral immunity. Nat Immunol 13(3): 214-222.

24. Kobari L, Pflumio F, Giarratana M, Li X, Titeux M, et al. (2000) In vitro- and in vivo-evidence for the longterm multilineage (myeloid, B-, NK, and T) reconstitution capacity of ex vivo-expanded human CD34(+) cord blood cells. Exp. Hematol., 28(12): $1470-1480$.

25. Kyba M, Perlingeiro RC, Hoover RR, Lu CW, Pierce J, et al. (2003) Enhanced hematopoietic differentiation of embryonic stem cells conditionally expressing Stat5. Proc Natl Acad Sci USA 100(1): 11904-11910.

26. Sainova IV, Vavrek I, Pavlova V, Daneva T, Iliev I, et al. (2011) Experimental model of safe in vitro- and in vivo-influence of internal and external factors of cell differentiation. Afr J Pharm Pharmacol 5(6): 786-791.

27. Brachmann CB, Davies A, Cost GJ, Caputo E, Li J, et al. (1998) Designer deletion strains derived from Sacharomyces cerevisiae S288C: a useful set of strains and plasmids for PCR-mediated gene disruption and other applications. Yeast 14(2): 115132.

28. Wang M, Bronte V, Chen PW, Gritz L, Panicali D, et al. (1995) Active immunotherapy of cancer with non-replicating recombinant fowlpox virus encoding a model tumor-associated antigen. J Immunol 154(9): 4685-4692.

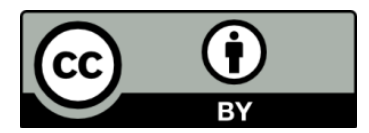

Sainova IV, et al. Development of Methods for Safe Application of Viral Vectors for Production of Gene-Engineering Vaccines. Virol Immunol J 2018, 2(7): 000176. 